\title{
HEMIPELVECTOMIA: EXPERIÊNCIA DO HOSPITAL ERASTO GAERTNER COM 32 CASOS EM 10 ANOS
}

\author{
HEMIPELVECTOMY: ERASTO GAERTNER HOSPITAL'S EXPERIENCES \\ WITH 32 CASES IN TOYEARS
}

Rosyane Rena de Freitas', André Luiz Soares Crivellaro', Glauco José Pauka Mello², Mário Armani Neto², Geraldo de Freitas Filho², Letícia Viani da Silva ${ }^{3}$

\section{RESUMO}

Objetivo: Mostrar a experiência do Hospital Erasto Gaertner com as cirurgias de hemipelvectomias em um período de 10 anos. Métodos: Estudo retrospectivo de 32 pacientes submetidos à hemipelvectomia de 1998 a 2008, avaliando características clínico-cirúrgicas. Resultados: Dos 32 pacientes, 15 eram do sexo feminino e 17 do masculino. A média de idade foi de 37,94 anos. Oito casos apresentavam comprometimento de feixe vasculonervoso: três localizavam-se em ilíaco com extensão para a coxa, dois em acetábulo com extensão para coxa e três em acetábulo e púbis. Vinte e três apresentavam o feixe vasculonervoso livre de neoplasia: 11 restritos ao ilíaco, seis em região acetabular, dois em ramo púbico, quatro com extensão a toda hemipelve óssea. Um apresentava comprometimento de vasos ilíacos-femorais: um em ramo púbico. Sete casos de condrossarcoma e quatro de sarcoma de Ewing, representaram a maioria. Oito foram submetidos à hemipelvectomia externa e 24 à hemipelvectomia interna (11 tipo I, quatro tipo II, dois tipo II + III, três tipo III e quatro tipo IV). Destes 24 casos, 13 sem reconstrução, 10 com enxerto de fíbula e um com prótese de veia e artéria ilíaco-femorais. Vinte e seis cirurgias foram curativas e seis paliativas. Houve 14 óbitos. Sobrevida de dois e cinco anos observada em 11 e 10 casos, respectivamente. Seis casos apresentam menos de dois anos de cirurgia. Em três casos houve perda de acompanhamento. Conclusão: Os dados mostram a experiência de um serviço de referência em oncologia, especializado no tratamento de cirurgias de alta complexidade.

Descritores - Hemipelvectomia; Neoplasias pélvicas; Pelve

\section{ABSTRACT}

Objective: To review the experience of the Erasto Gaertner Hospital with hemipelvectomies of surgeries over a period of 10 years. Methods: A retrospective study of 32 patients who underwent hemipelvectomy in Erasto Gaertner Hospital from 1998 to 2008, assessing clinical and surgical characteristics. Results: Of 32 patients, 15 were female and 17 male. The mean age was 37.94 years. Eight cases showed involvement of the neurovascular bundle: 3 were located in the iliac and extended to the thigh, 2 were in the acetabulum and extended to the thigh and 3 were in the acetabulum and pubis. One involved the iliac-femoral vessels: one in the pubic ramus. Seven cases of chondrosarcoma and 4 cases of Ewing's sarcoma represented the majority. Eight underwent external hemipelvectomy and 24 underwent internal hemipelvectomy (11 were type I; 4 were type II; 2 were type II + III; 3 were type III and 4 were type IV). Of these 24 cases, 13 were without reconstruction, 10 were with a fibular graft and 1 was with an iliac-femoral vein and artery prosthesis. Twenty-six surgeries were curative and 6 palliative. There were 14 deaths. Survival of 2 and 5 years were seen in 11 and 10 cases, respectively. Six cases have less than 2 years of surgery. Three cases were lost during follow-up. Conclusion: The study shows the experiences of an exemplary oncology service, specializing in highly complex surgical treatment.

Keywords - Hemipelvectomy; Pelvic neoplasms; Pelvis

\footnotetext{
1 - Residentes de Cirurgia Oncológica do Hospital Erasto Gaertner, Curitiba, PR.

2 - Ortopedistas do Serviço de Ortopedia Oncológica do Hospital Erasto Gaertner, Curitiba, PR.

3 - Acadêmica de Medicina da PUC-PR.
}

Trabalho realizado Hospital Erasto Gaertner e Liga Paranaense de Combate ao Câncer - Curitiba, PR.

Correspondência: Travessa Frei Caneca, 105, Ap. 44, Centro - 80010-090 - Curitiba, PR. E-mail: rosyanerf@yahoo.com.br 


\section{INTRODUÇÃO}

Os ossos da região pélvica são sede de menos de $5 \%$ de todos os tumores ósseos malignos. Apesar desta raridade, constituem capítulo à parte no tratamento dos tumores ósseos, devido à complexidade anatômica da região. Permanecem, portanto, sendo um problema de difícil solução, ainda nos dias de hoje $\mathrm{e}^{(1,2)}$.

A hemipelvectomia externa (ressecção de toda a hemipelve, incluindo o membro inferior) é especialmente indicada em pacientes com tumores extensos, comprometendo a hemipelve com envolvimento da raiz da coxa. Com o desenvolvimento de novas drogas quimioterápicas, radioterapia, métodos diagnósticos e novas técnicas cirúrgicas, houve aumento do número de pacientes que foram submetidos a cirurgias de preservação dos membros (hemipelvectomia interna) ${ }^{(1-4)}$. Desde que seja possível boa margem oncológica de ressecção, sem amputação, as cirurgias preservadoras estão indicadas, no sentido de obter resultados oncológicos semelhantes àqueles obtidos pela hemipelvectomia externa. Ocasionalmente, a hemipelvectomia com intenção paliativa é realizada a fim de proporcionar controle local, quando outras modalidades terapêuticas menos agressivas como radioterapia, quimioterapia, antibióticos, analgésicos potentes, entre outras, indicadas previamente não determinarem o efeito desejado ${ }^{(3)}$.

O objetivo deste estudo foi mostrar a experiência do Hospital Erasto Gaertner de Curitiba com as cirurgias da cintura pélvica de 1998 a 2008.

\section{PACIENTES E MÉTODOS}

Foram estudados retrospectivamente 32 pacientes submetidos à hemipelvectomia no Hospital Erasto Gaertner em Curitiba no período de 1998 a 2008 e avaliadas características clínicas e cirúrgicas.

\section{RESULTADOS}

Dos 32 pacientes, a idade mínima foi de nove anos, a máxima de 73 e a média de 37,94 anos.

Quinze casos eram do sexo feminino e 17, do masculino.

Oito casos apresentavam invasão tumoral do feixe vasculonervoso: três localizavam-se em ilíaco com extensão para a coxa, dois em acetábulo com extensão para coxa e três em acetábulo e púbis (Figura 1). Vinte e três não apresentavam invasão tumoral do feixe vasculonervoso: 11 restritos ao ilíaco, seis em região acetabular, dois em ramo púbico, quatro com extensão em toda hemipelve óssea (Figura 2). Uma paciente apresentava tumor em região do púbis com comprometimento da artéria e veia femorais sem comprometimento neural.

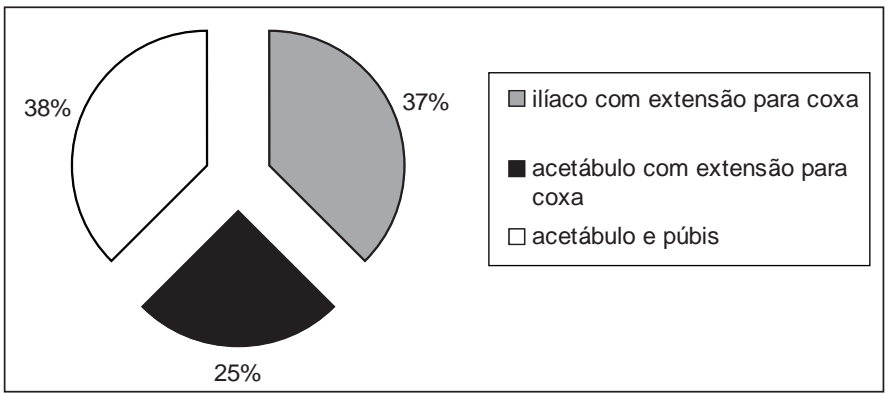

Figura 1 - Localização dos tumores com comprometimento do feixe vasculonervoso

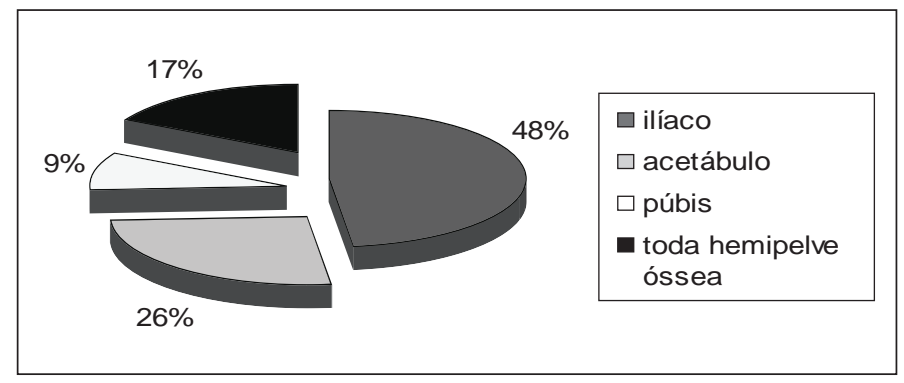

Figura 2 - Localização dos tumores sem comprometimento do feixe vasculonervoso

O menor diâmetro do tumor foi de $8 \mathrm{~cm}$, o máximo de $30 \mathrm{~cm}$ com média de $16,13 \mathrm{~cm}$.

Com relação ao tipo histológico, a maioria (sete casos ) foi representada por condrossarcoma, em segundo lugar, sarcoma de Ewing (quatro casos). Os demais tipos histológicos estão descritos no Quadro 1.

Quadro 1 - Histopatologia

\begin{tabular}{|l|l|}
\hline \multicolumn{1}{|c|}{ Tipo histológico } & N \\
\hline Adenocarcinoma & 2 \\
\hline Carcinoma espinocelular & 1 \\
\hline Condrossarcoma & 7 \\
\hline Fibrohistiocitoma maligno & 3 \\
\hline Hemangiossarcoma & 1 \\
\hline Osteossarcoma & 3 \\
\hline PNET & 2 \\
\hline Rabdomiossarcoma alveolar & 1 \\
\hline Rabdomiossarcoma embrionário & 1 \\
\hline Sarcoma de Ewing & 4 \\
\hline Sarcoma pleomórfico e fusocelular & 2 \\
\hline Sarcoma sinovial & 3 \\
\hline Tumor de células gigantes & 2 \\
\hline Total & 32 \\
\hline
\end{tabular}

Dos 32 pacientes, oito casos (25,1\%) foram submetidos à hemipelvectomia externa: seis com reconstrução utilizando retalho glúteo posterior e dois com retalho miocutâneo anterior da coxa (Figuras 3 e 4). 
Vinte e quatro casos (75\%) foram submetidos à hemipelvectomia interna, assim distribuídos de acordo com a classificação de Enneking: 11 tipo I, quatro tipo II, dois tipo II + III, três tipo III e quatro tipo IV (Figura 5).

Dos 24 casos submetidos à hemipelvectomia interna, 13 (54\%) não tiveram reconstrução, 10 (42\%) foram submetidos a colocação de enxerto de fíbula e em um caso (4\%) foi necessário enxerto de prótese vascular de veia e artéria ilíaco-femorais. Dos 10 casos submetidos a enxerto de fíbula, quatro foram submetidos à hemipelvectomia tipo IV, dois tipo II + III e quatro tipo II (Figura 6).

A paciente submetida a enxerto de vasos ilíaco-femorais apresentava tumoração com infiltração direta de artéria e veia ilíacas-femorais, sem comprometimento nervoso, sendo submetida a hemipelvectomia tipo III com ressecção em bloco destes vasos junto ao tumor e reconstrução utilizando próteses de PTFE em veia e artéria.

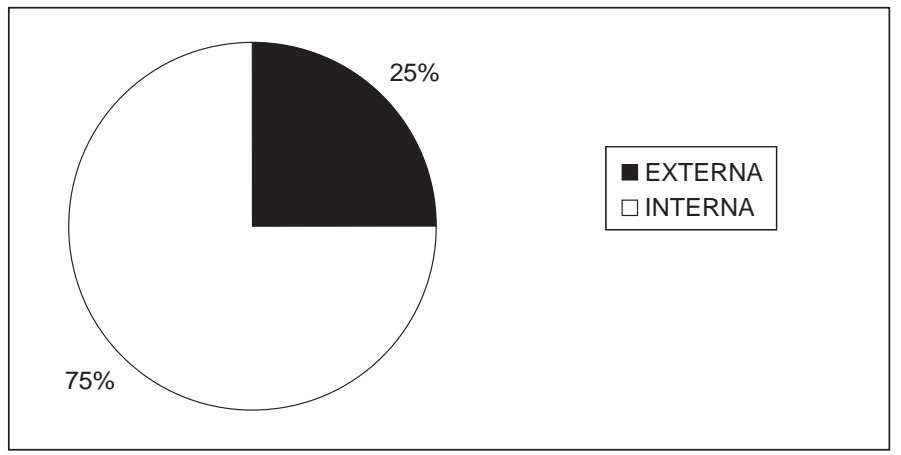

Figura 3 - Tipo de hemipelvectomia

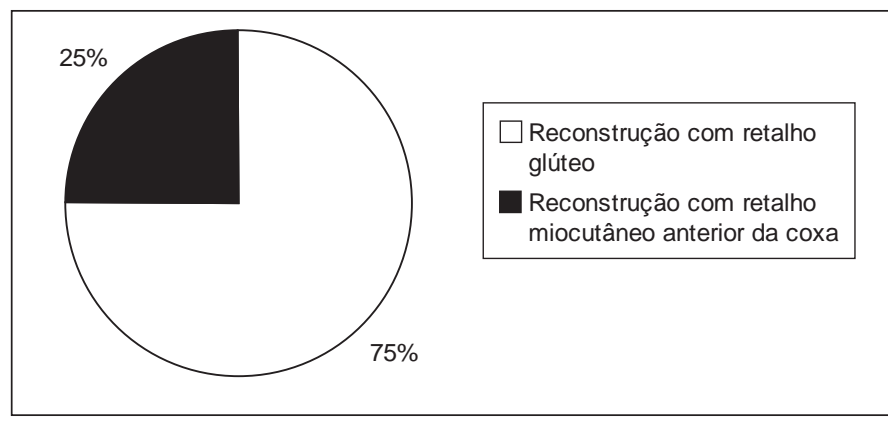

Figura 4 - Tipo de reconstrução em hemipelvectomia externa

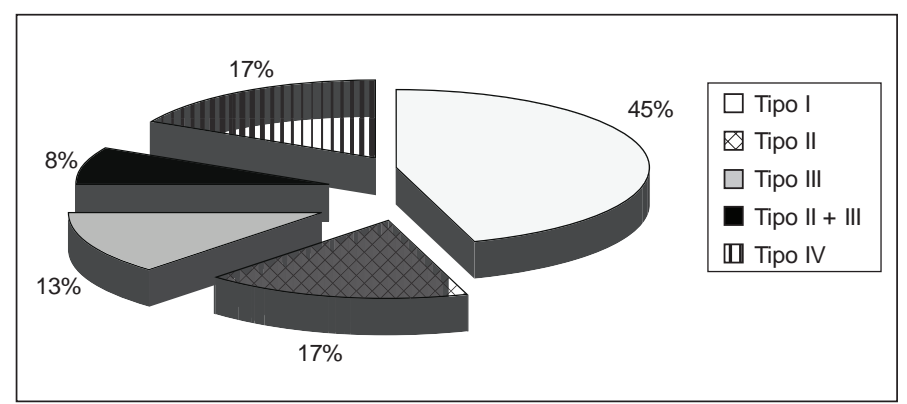

Figura 5 - Tipo de hemipelvectomia interna

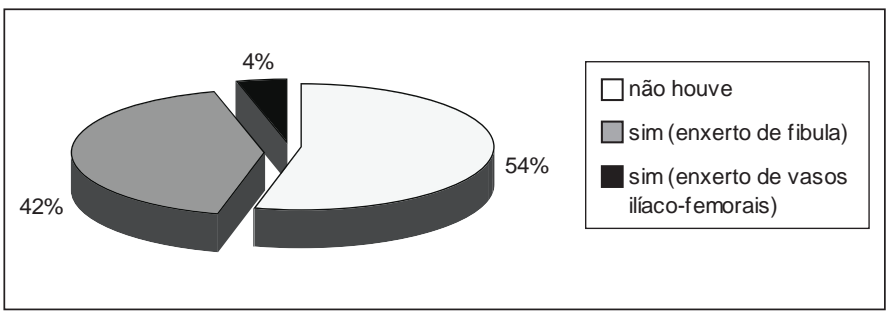

Figura 6 - Reconstrução em hemipelvectomia interna

Dos 32 pacientes, 26 casos (81,3\%) foram operados com intenção curativa e seis $(18,8 \%)$ com intenção paliativa (Figura 7).

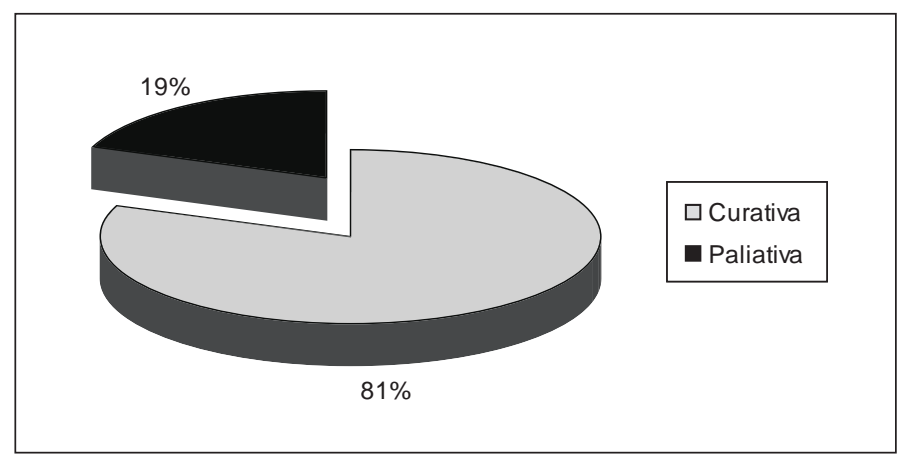

Figura 7 - Intenção curativa x paliativa

Em 24 casos (75\%) a cirurgia não complicou, em cinco (15,6\%) houve infecção de ferida operatória, em dois $(6 \%)$ hematoma e em um caso $(3,1 \%)$, necrose parcial de enxerto (Figura 8).

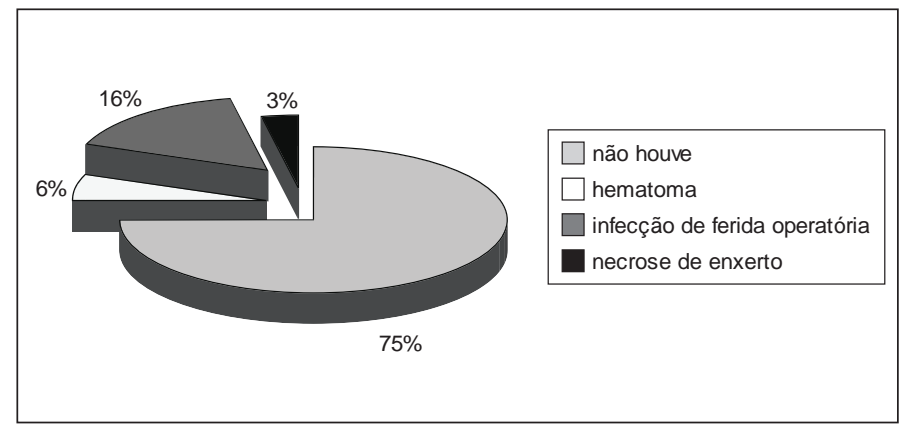

Figura 8 - Complicações

No presente estudo não foi evidenciada recorrência local. Recorrência à distância foi de 21,8\% (Quadro 2).

Com relação às margens cirúrgicas, 12,5\% apresentaram margens microscópicas comprometidas (ressecção R1). Todos estes casos foram submetidos a tratamento adjuvante com radioterapia e quimioterapia, não sendo submetidos a nova intervenção cirúrgica. Os tipos de hemipelvectomia, a sobrevida e o tipo histológico destes tumores estão especificados no Quadro 3. 
Quadro 2 - Óbito em pacientes com recorrência à distância

\begin{tabular}{|l|l|}
\hline Um caso em seis meses & PULMÃo - Óbito em um ano \\
\hline Um caso em cinco anos & PULMÃo - Óbito em cinco anos \\
\hline Dois casos em um ano & $\begin{array}{l}\text { PULMÃo - Uma perda do acompanhamento; } \\
\text { óbo em dois anos }\end{array}$ \\
\hline Um caso em sete meses & PULMÃo - Óbito em um ano \\
\hline Um caso em dois meses & COLUNA - Óbito em três meses \\
\hline Um caso em quatro meses & COLUNA - Óbito em cinco meses \\
\hline
\end{tabular}

Quadro 3 - Tipos de hemipelvectomia, sobrevida e tipo histológico dos tumores com ressecção $\mathrm{R} 1$

\begin{tabular}{|l|l|l|l|}
\hline Um caso & $\begin{array}{l}\text { Hemipelvectomia } \\
\text { interna tipo I }\end{array}$ & Óbito em um ano & Sarcoma de Ewing \\
\hline Um caso & $\begin{array}{l}\text { Hemipelvectomia } \\
\text { interna tipo IV }\end{array}$ & Óbito em cinco meses & Condrossarcoma \\
\hline Um caso & $\begin{array}{l}\text { Hemipelvectomia } \\
\text { interna tipo I }\end{array}$ & $\begin{array}{l}\text { Perda de seguimento } \\
\text { após oito anos }\end{array}$ & Condrossarcoma \\
\hline Um caso & $\begin{array}{l}\text { Hemipelvectomia } \\
\text { interna tipo I }\end{array}$ & $\begin{array}{l}\text { Cirurgia em dois } \\
\text { meses }\end{array}$ & Fibrohistiocitoma maligno \\
\hline
\end{tabular}

No período estudado houve 14 casos (44\%) de óbito com três casos (9\%) de perda de acompanhamento (Figura 9). O tempo mínimo de sobrevida foi de um dia, máximo de cinco anos e média de 10,6 meses. Destes 14 óbitos, quatro eram de pacientes submetidos à cirurgia paliativa e morreram por consequência direta da doença (Quadro 4). Os outros 10 pacientes foram a óbito por causa não relacionada à intervenção cirúrgica, sendo que nenhuma complicação cirúrgica relatada apresentou impacto na sobrevida dos pacientes.

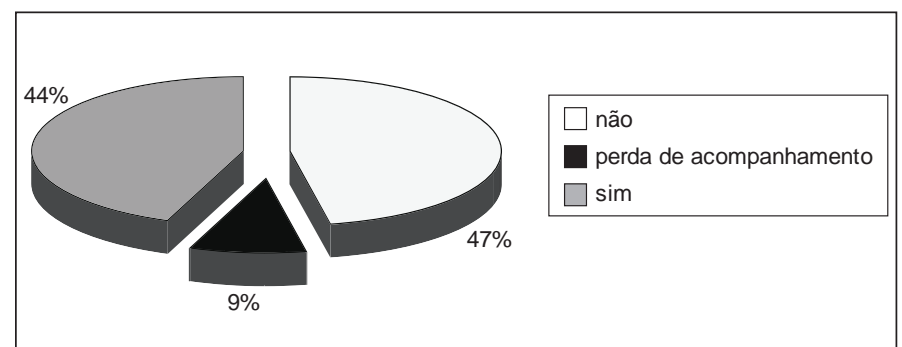

Figura 9 - Óbito

Quadro 4 - Sobrevida em pacientes submetidos à hemipelvectomia paliativa

\begin{tabular}{|l|l|}
\hline Um caso & Um dia \\
\hline Um caso & Dois meses \\
\hline Um caso & Três meses \\
\hline Um caso & Cinco meses \\
\hline Dois casos & Perda de acompanhamento \\
\hline
\end{tabular}

Sobrevida em dois anos foi observada em 11 casos $(34,4 \%)$, seis casos $(18,8 \%)$ com cirurgia recente (menos de dois anos de acompanhamento) e três casos (9,4\%) perderam o acompanhamento (Figura 10). Nenhum caso com recorrência à distância teve sobrevida em dois anos.

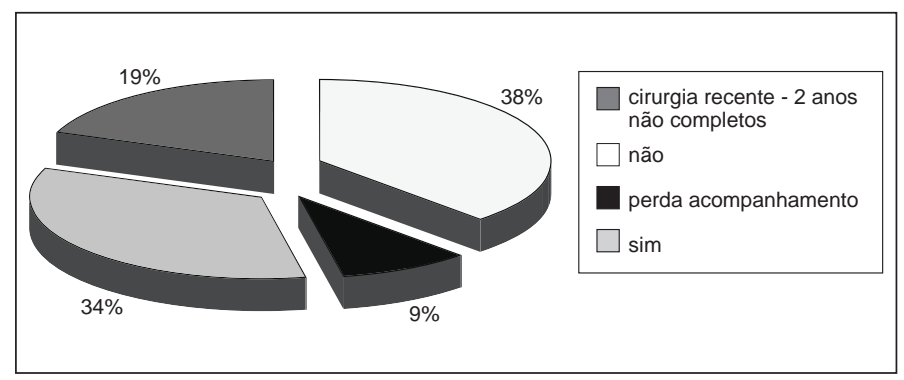

Figura 10 - Sobrevida em dois anos

Sobrevida em cinco anos foi observada em 10 casos $(31,3 \%)$, seis casos $(18,8 \%)$ com cirurgia recente (menos de cinco anos de acompanhamento) e três casos $(9,4 \%)$ perderam o acompanhamento (Figura 11). Nenhum caso com recorrência à distância teve sobrevida em cinco anos.

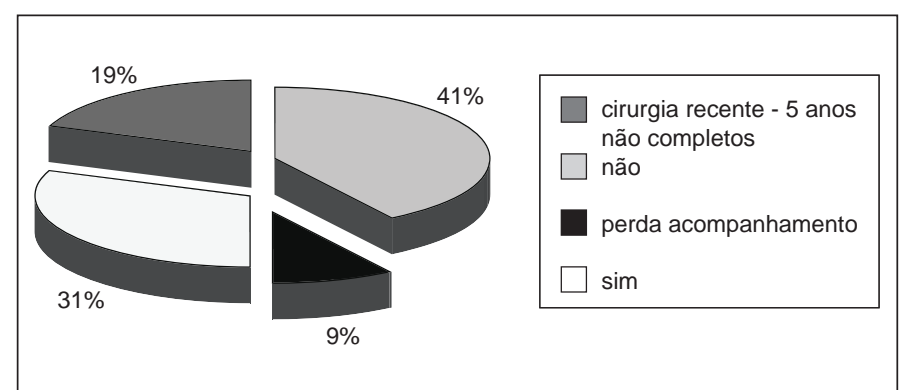

Figura 11 - Sobrevida em cinco anos

Com relação ao ponto de vista funcional dos pacientes submetidos à hemipelvectomia, os 15 casos sobreviventes foram submetidos à entrevista recente para este estudo, a fim de determinar a satisfação do paciente com a cirurgia, sendo o resultado final baseado no estadiamento funcional segundo Enneking, adotado pela Musculoskeletal Tumor Society que considera:

1 -Excelente: sem dor, função sem restrições e ótima aceitação do paciente;

2 - Bom: dor de pouca intensidade não incapacitante e/ou restrição à função de recreação e boa aceitação do paciente; 3 -Regular: dor moderada com incapacidade intermitente e/ou parcial restrição ocupacional e regular aceitação do paciente;

4 - Ruim: dor de grande intensidade com contínua incapacidade, e/ou total restrição ocupacional e paciente insatisfeito. 
Os dados obtidos são evidenciados na Figura 12.

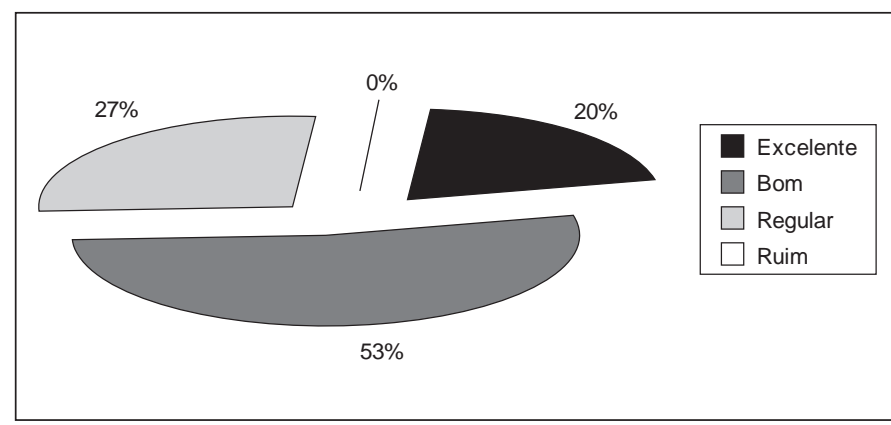

Figura 12 - Resultado funcional global, de acordo com a classificação categórica de Enneking et al (1993), em pacientes submetidos à hemipelvectomia

Nas Figuras 13, 14 e 15 observamos, respectivamente: tempos cirúrgicos evidenciando reconstrução utilizando a fíbula em hemipelvectomia interna tipo IV; reconstrução vascular de artéria e veia ilíacas-femorais em hemipelvectomia interna; e o aspecto final dos membros inferiores (MMII) da paciente submetida à reconstrução vascular.

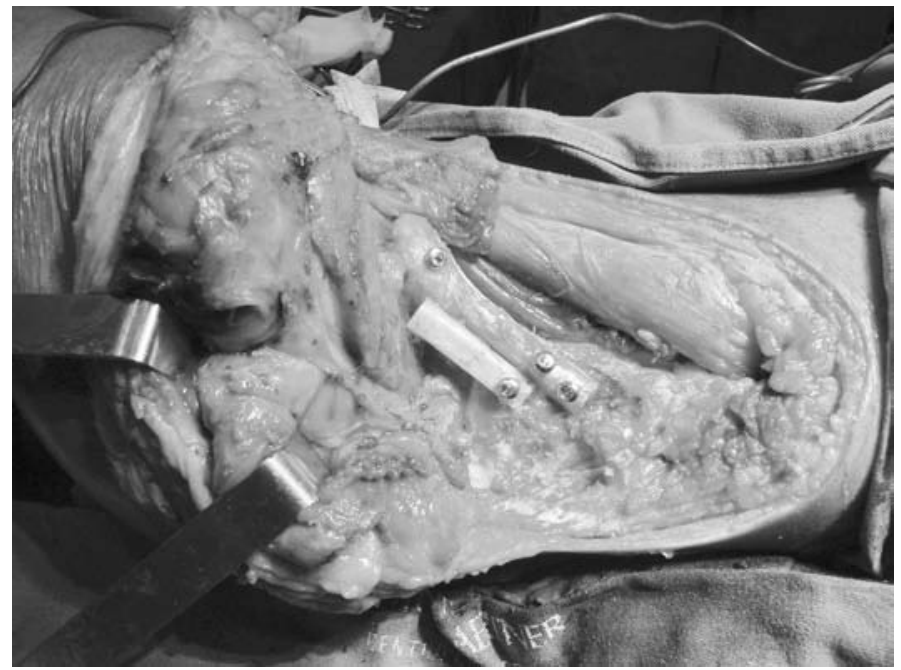

Figura 13 - Reconstrução utilizando a fíbula em hemipelvectomia interna tipo IV

\section{DISCUSSÃO}

Apesar de todo o desenvolvimento da cirurgia oncológica, a ressecção dos tumores pélvicos é um dos problemas que mais têm sofrido mudanças nas cirurgias preservadoras do membro.

Os tumores malignos mais comumente encontrados na região pélvica, em ordem de frequência, são o condrossarcoma, o sarcoma de Ewing e o osteossarcoma ${ }^{(3)}$. Em nosso estudo, a maioria foi constituída por condrossarcoma, em segundo lugar, sarcoma de Ewing; dados concordantes com a literatura.

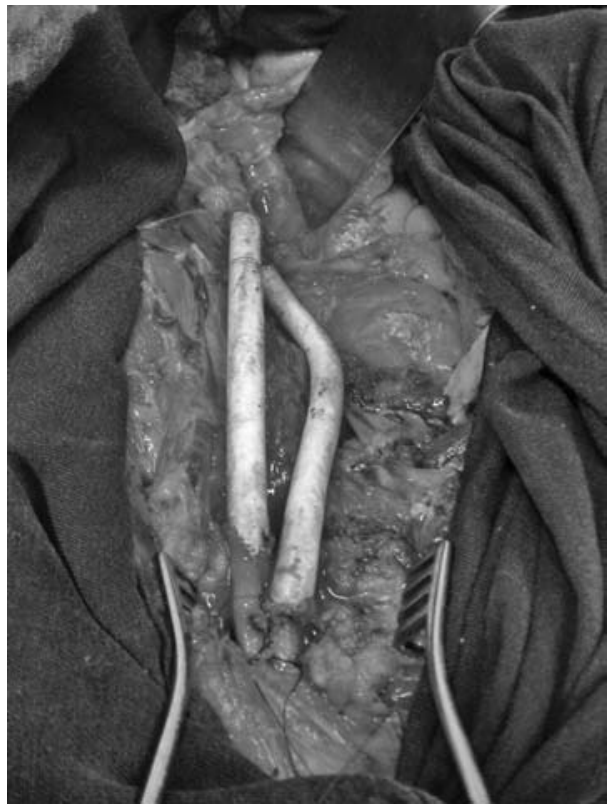

Figura 14 - Reconstrução vascular de artéria e veia ilíacasfemorais em hemipelvectomia interna

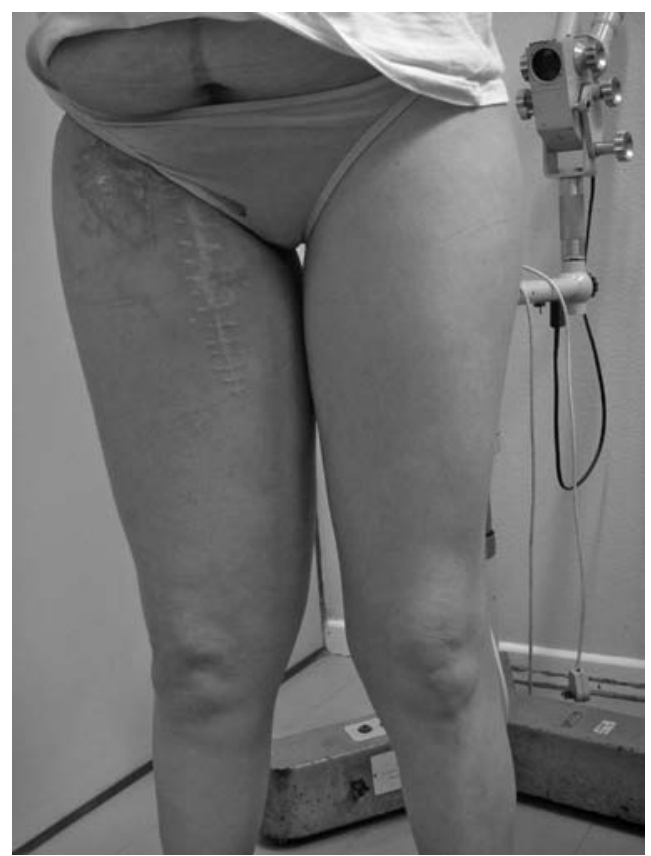

Figura 15 - Aspecto final dos MMII da paciente submetida à reconstrução vascular

O tipo de cirurgia (hemipelvectomia externa ou interna) está baseado no tamanho do tumor e/ou no local das estruturas e tecidos adjacentes envolvidos ${ }^{(5)}$. Tumores extensos da região pélvica invadindo osso, partes moles, nervos e vasos são difíceis de tratar conservadoramente. A hemipelvectomia externa é um procedimento de morbidade considerável, sendo atualmente indicada em pequena parcela dos tumores pélvicos, quando a extensão do tumor, o comprometimento da raiz da coxa e feixe vasculoner- 
voso impossibilitam a preservação do membro. Nestes casos, pode ser a única alternativa para a ressecção de tais tumores com margens adequadas e com um intervalo livre de doença aceitável. Em uma hemipelvectomia externa padrão, o retalho miocutâneo glúteo é o mais utilizado. Para extensos tumores glúteos ou da região proximal posterior da coxa que necessitam hemipelvectomia para tratamento, o retalho miocutâneo anterior da coxa, mantido pelos vasos femorais é, sem dúvida, a opção mais apropriada e segura $^{(6,7)}$. Em nosso estudo, dos 32 pacientes, oito $(25,1 \%)$ foram submetidos à hemipelvectomia externa (seis com retalho glúteo e dois miocutâneo anterior da coxa).

A hemipelvectomia interna é um procedimento complexo, mas cosmeticamente superior à hemipelvectomia externa. É indicada em tumores menores, restritos à hemipelve, sem comprometimento da coxa e sem invasão neurovascular do feixe iliofemoral. Nos casos em que houver invasão vascular, sem comprometimento neural, a cirurgia pode ser indicada com reconstrução da artéria e/ou veia com prótese vascular, a fim de manter funcional o membro inferior ipsilateral ${ }^{(8)}$. Em nosso estudo, um dos casos foi submetido à reconstrução vascular associada.

O tipo de hemipelvectomia interna segundo a classificação de Enneking, baseia-se na localização do tumor na pelve: tipo I (asa do ilíaco), tipo II (região periacetabular), tipo III (arco púbico) e tipo IV (toda hemipelve óssea). Em nosso estudo, 24 casos (75\%) foram submetidos à hemipelvectomia interna: 11 casos tipo I, dois tipo II + III, quatro tipo II, três tipo III e quatro tipo IV.

Ponto ainda controverso na literatura é a questão da reconstrução nas hemipelvectomias internas. Em um estudo avaliando 31 casos - 12 e 19 pacientes retrospectivamente nos hospitais AC Camargo (São Paulo) e Boldrini (Campinas) - submetidos à hemipelvectomia tipo II, com ou sem reconstrução do anel pélvico utilizando a fíbula, os pacientes submetidos à hemipelvectomia interna com reconstrução do anel pélvico com autoenxerto de fíbula tiveram melhor resultado funcional global comparativamente ao grupo dos que não foram submetidos à reconstrução $(\mathrm{p}=0,007)^{(9)}$. Em nosso estudo, dos 24 casos submetidos à hemipelvectomia interna, 10 (42\%) foram submetidos à colocação de enxerto de fíbula: quatro tipo IV, dois tipos II + III e quatro tipo II. Não foi realizado em nosso serviço, estudo funcional comparativo entre os dois grupos.

Na literatura, podem ser observadas duas correntes não consensuais em relação à indicação da reconstrução pélvica após a hemipelvectomia interna:

- Os cirurgiões que não indicam a reconstrução pélvica ressaltam a dificuldade da abordagem cirúrgica da pelve, por ser próxima a órgãos e feixes vasculonervosos. Guest et $a l^{(10)}$ referiram que a reconstrução do acetábulo é tão difícil quanto a remoção do tumor. Veth et $a l^{(11)}$ reforçaram o fato de que após a ressecção do tumor pélvico a função do quadril e do membro inferior nunca seriam normais, independente da reconstrução realizada. Estes autores publicaram resultados funcionais satisfatórios em pacientes sem a reconstrução. Outro aspecto considerado por Hugate e $\operatorname{Sim}^{(12)}$ foi o tempo de cirurgia e a perda sanguínea, que são menores em cirurgias sem reconstrução. É importante fazer a ressalva de que em pacientes que realizam ressecções do tipo I (lesão da asa do ilíaco) ou do tipo III (lesão em região isquiopúbica) não é necessária a reconstrução, pois a alteração biomecânica ocasionada não acarreta perda funcional considerável ${ }^{(12)}$.

Os cirurgiões que defendem a reconstrução justificam o fato pela possibilidade de restaurar a estabilidade pélvica, manter a mobilidade do quadril, minimizar a discrepância do comprimento dos membros inferiores e, consequentemente, obter melhor resultado funcional e estético ${ }^{(13,14)}$.

Contudo, ainda não existe consenso em relação a reconstruir ou não a pelve após a ressecção do tumor. Não está definido qual tipo de cirurgia traz maiores benefícios ao paciente. Vários estudos têm sido realizados nas últimas décadas e cada um apresenta vantagens e desvantagens das diversas técnicas cirúrgicas relacionadas à ressecção de tumores pélvicos.

Alguns outros aspectos também continuam controversos. O primeiro é o controle da doença e sobrevida pós-cirúrgica. Estudos mostram que, após ressecções com hemipelvectomia para sarcomas de alto grau, a taxa de sobrevida relatada varia até $40 \%$, com recidiva local de $70 \%(8,15)$. Em nosso trabalho, considerando os diversos tipos histológicos, o tempo médio de sobrevida foi de 10,6 meses, sobrevida em dois e cinco anos de 34,4\% e 31,3\%, respectivamente. Recidiva local, uma das principais complicações, com importante impacto na sobrevida, não foi observada neste estudo. O segundo aspecto é a alta taxa de complicações relatadas nestas extensas e complexas cirurgias, tais como infecções superficiais e profundas, seromas, deiscências de sutura, soltura dos enxertos de fíbula, neuropraxias e trombose venosa entre as mais comuns, sendo que infecção e lesão nervosa, entre outras, atingem incidência de $50 \%{ }^{(5,8,16)}$. Em nosso estudo, em $75 \%$ dos pacientes, a cirurgia não complicou; em 15,6\% houve infecção superficial de ferida operatória; em 6\%, hematoma superficial em subcutâneo; e em 3,1\%, necrose parcial de enxerto. Nenhuma destas complicações necessitou de nova intervenção cirúrgica, sendo tratadas com antibioticoterapia, drenagem ambulatorial de hematoma superficial em ferida operatória e desbridamento de bordas 
de ferida operatória respectivamente. Não foram observados casos de soltura de enxertos de fíbula, neuropraxias severas, trombose ou outras complicações mais severas.

Hemipelvectomia paliativa tem sido indicada em pacientes com doença óssea metastática ou localmente avançada $^{(17,18)}$. Indicações incluem dor intratável, ulceração, hemorragia, infecção e fratura patológica instável; porém, ainda não há consenso na literatura sobre essa indicação. Devido ao fato de a hemipelvectomia paliativa ser controversa, especialmente em casos com menos de um ano de sobrevida, é de suma importância avaliar outras opções antes da cirurgia ${ }^{(17,18)}$. Em nosso estudo, seis pacientes foram operados com intenção paliativa. Destes, a sobrevida foi de um, 60, 90 e 180 dias nos pacientes que seguiram acompanhamento, sendo que em dois casos houve perda de seguimento, não sendo, portanto, possível determinar o tempo de sobrevida. A análise final da melhora da qualidade de vida obtida com a cirurgia não foi quantificada através de questionários padronizados. Dessa forma, devido ao desenho retrospectivo deste estudo não conseguimos concluir sobre os resultados atingidos com a indicação paliativa. No entanto, vale ressaltar que as indicações foram baseadas na sintomatologia do paciente com piora da dor, infecção e sangramento local não responsivo a tratamento medicamentoso e radioterapia, com desejo cirúrgico referido pelo paciente, o qual foi conscientizado da impossibilidade curativa e da extensão do procedimento.

Com relação aos resultados funcionais, em vários ca-

\section{REFERÊNCIAS}

1. Campanacci M, Capanna R. "Reconstruction for periacetabular pelvic resection: closing remarks". In: Enneking WF. Limb salvage in musculoskeletal oncology. New York: Churchill-Livingstone; 1987. p. 181-91.

2. Campanacci M, Capanna R. Pelvic resections: the Rizzoli Institute experience. Orthop Clin North Am. 1991;22(1):65-86.

3. Eilber FR, Eckardt JJ, Grant TG. "Resection of malignant bone tumors of the pelvis": evaluation of local recurrence, survival, and function". In Enneking WF. Limb salvage in musculoskeletal oncology. New York: Churchill-Livingstone; 1987. p.136-41.

4. Enneking WF, Dunham WK. Resection and reconstruction for primary neoplasms involving the innominate bone. J Bone Joint Surg Am. 1978;60(6):731-46.

5. Ham SJ, Schraffordt Koops H, Veth RP, van Horn JR, Eisma WH, Hoekstra HJ. External and internal hemipelvectomy for sarcomas of the pelvic girdle: consequences of limb-salvage treatment. Eur J Surg Oncol. 1997;23(6):540-6.

6. Corrêa DS, Rossi BM, Ferreira FO, Nakagawa WT, Guimarães GC, Lopes A. Reconstrução com retalho miocutâneo anterior da coxa após hemipelvectomia por carcinoma de células escamosas de região glútea: relato de caso. Rev Bras Cancerol. 2002;48(2):253-6.

7. Vieira LJ, Vieira JP, Oliveira AF, Freitas RR. Hemipelvectomy utilizing an anterior myocutaneous flap: case report and surgical technique description. Rev Bras Cancerol. 2004;50(4):301-5.

8. Apffelstaedt JP, Driscoll DL, Spellman JE, Velez AF, Gibbs JF, Karakousis CP. Complications and outcome of external hemipelvectomy in the management of pelvic tumors. Ann Surg Oncol. 1996;3(3):304-9.

9. Deneno BP, Gonçalves JCB, Lopes A, Brandalise SR. Resultado funcional em crianças e adolescentes submetidos à hemipelvectomia interna tipo II, com ou sem reconstrução do anel pélvico. Rev Bras Ortop. 2007;42(5):125-32.

10. Guest CB, Bell RS, Davis A, Langer F, Ling H, Gross AE, Czitrom A. Allograftimplant composite reconstruction following periacetabular sarcoma resection. J Arthroplasty. 1990;5 Suppl:S25-34. sos, eles são bons e animadores, permitindo inclusive que alguns pacientes caminhem sem auxílio de muletas ou bengalas, possibilitando também apoio monopodálico com carga. Alguns deles necessitam de vários meses para a sua reabilitação, obtendo condições de marcha com auxílio de muletas, e outros conseguem deambular sem nenhum tipo de órtese ${ }^{(17-20)}$. No entanto, devido ao caráter retrospectivo da maioria dos estudos, é difícil de quantificar e qualificar o grau de controle dos sintomas atingidos. A avaliação funcional neste estudo foi realizada através do estadiamento funcional segundo Enneking( ${ }^{(21)}$, adotado pela Musculoskeletal Tumor Society. Através desta classificação, dos 15 pacientes sobreviventes arguidos recentemente para este estudo sobre a função do membro operado, três referiam resultados excelentes, oito bons e quatro regulares. Estes quatro últimos referiram resultados regulares pela necessidade do uso de muletas em menos de 50\% do tempo para realizar suas atividades habituais.

Em conclusão, este estudo mostrou que a hemipelvectomia é cirurgia viável, devido ao baixo índice de complicações pós-operatórias e à ausência de recidiva local em nossa amostra, aspectos evidenciados em porcentagem inferior à literatura. O tipo de hemipelvectomia indicado depende essencialmente da extensão local da doença. Para pacientes cuja cura não é esperada, estudos prospectivos sobre a qualidade de vida são necessários para validar esta cirurgia, considerando o desejo do paciente, as condições locais do tumor e os tratamentos prévios realizados.

11. Veth RP, Schraffordt Koops H, Nielsen HK, Oldhoff J, Verkerke GJ, Postma A. A critique of techniques for reconstruction after internal hemipelvectomy for osteosarcoma. Cancer Treat Res. 1993;62:221-9.

12. Hugate R Jr, Sim FH. Pelvic reconstruction techniques. Orthop Clin North Am. 2006;37(1):85-97.

13. Marchese VG, Ogle S, Womer RB, Dormans J, Ginsberg JP. An examination of outcome measures to assess functional mobility in childhood survivors of osteosarcoma. Pediatr Blood Cancer. 2004;42(1):41-5.

14. Pring ME, Weber KL, Unni KK, Sim FH. Chondrosarcoma of the pelvis. A review of sixty-four cases. J Bone Joint Surg Am. 2001;83(11):1630-42.

15. Renard AJ, Veth RP, Schreuder HW, Pruszczynski M, Keller A, van Hoesel Q et al. The saddle prosthesis in pelvic primary and secondary musculoskeletal tumors: functional results at several postoperative intervals. Arch Orthop Trauma Surg. 2000;120(3-4):188-94.

16. Apffelstaedt JP, Driscoll DL, Karakousis CP. Partial and complete internal hemipelvectomy: complications and long-term follow-up. J Am Coll Surg. 1995.

17. Malawer MM, Buch RG, Thompson WE, Sugarbaker PH. Major amputations done with palliative intent in the treatment of local bony complications associated with advanced cancer. J Surg Oncol. 1991;47(2):121-30.

18. Merimsky O, Kollender Y, Inbar M, Chaitchik S, Meller I. Palliative major amputation and quality of life in cancer patients. Acta Oncol. 1997;36(2):151-7.

19. Karakousis CP, Emrich LJ, Driscoll DL. Variants of hemipelvectomy and their complications. Am J Surg. 1989;158(5):404-8.

20. King LA, Downey GO, Savage JE, Twiggs LB, Oakley GJ, Prem KA. Resection of the pubic bone as an adjunct to management of primary, recurrent, and metastatic pelvic malignancies. Obstet Gynecol. 1989;73(6):1022-6.

21. Enneking WF, Dunham W, Gebhardt MC, Malawar M, Pritchard DJ. A system for the functional evaluation of reconstructive procedures after surgical treatment of tumors of the musculoskeletal system. Clin Orthop Relat Res. 1993. 